\title{
Pro Service Public in der Medizin?
}

\section{Hans Stalder}

Prof. Dr. med., Mitglied der Redaktion

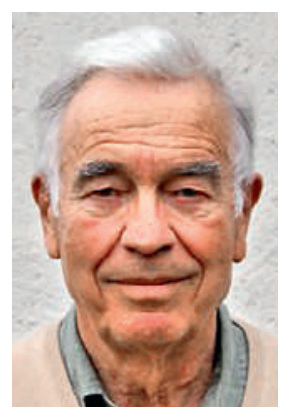

Zwar wurde die im Zusammenhang mit Post, SBB und Swisscom eingebrachte Volksinitiative Pro Service Public abgelehnt, doch sie führte immerhin zu einem vertieften Nachdenken über den Begriff «Service public» und erhitzte damit die Gemüter. Ich frage mich, ob das Gesundheitssystem als Service public par excellence eines Tages ebenfalls Gegenstand einer solchen Initiative werden könnte. Lassen sich die Argumente der Protagonisten der Initiative nicht auch auf die Gesundheit anwenden? Folgende Beschwerden waren vorgebracht worden:

Grundversorgung: Die Initiatoren gaben an, dass die Anzahl der Poststellen seit 1979 auf ein Drittel zurückgegangen und nur noch ein Viertel der SBB-Bahnhöfe in Betrieb seien.

In der Medizin beobachten wir zwischen 1980 und 2011 einen Rückgang der Spitalbetten um 35\%, ein Trend, der sich durch den nach der Einführung der DRG-Pauschale erwachsenden wirtschaftlichen Druck weiter verstärkt. Die durchschnittliche Dauer eines Spitalaufenthalts verringerte sich von 25,4 Tagen im Jahr 1982 auf 9,4 Tage 2011. Und auch der Zugang zu den ambulanten Diensten gestaltet sich immer prekärer. Immer weniger Allgemeinmediziner wollen sich in den ländlichen Regionen niederlassen. Bedingt durch Franchise und Kostenbeteiligung verzichteten 6,5\% der Schweizer aus Kostengründen auf den Arztbesuch (im Kanton Genf sind es 14,9\%, Zahnarztbesuche mit eingeschlossen). Vor allem Patienten mit polymorbiden Erkrankungen verzichten aus finanziellen Gründen auf die medizinische Behandlung; gerade sie müssten am dringendsten versorgt werden [1].

Kosten: Die Portogebühren sollen zwischen 1994 und 2015 um 62,5\%, das Bahnticket um 87\% gestiegen sein. Im Gesundheitswesen erhöhten sich die Prämien für die Grundversicherung in 14 Jahren um 50\%. Dies brachte viele in eine finanzielle Notlage, so dass mehr als ein Drittel der Bevölkerung staatlich unterstützt werden muss.

Unternehmenschefs werden immer besser bezahlt: Die Chefs von Post, SBB und Swisscom verdienen zwischen 72600 und 1832000 CHF. Und in der Medizin? Das lässt sich nicht so einfach ausfindig machen. So soll der Helsana-Chef beispielsweise 939000 CHF und der ehemalige Direktor der Groupe Mutuel über 2,2 Millionen pro Jahr verdiente haben ... Was die Vergütung der Leistungsträger in der Medizin, d.h. vor allem bei den Spi- talärzten, betrifft, beherrschen immer wieder sie die Leitartikel der Presse.

Investierte Gewinne: Die Befürworter der Initiative schlugen vor, die Gewinne aus dem Service public ausschliesslich in diesen zu reinvestieren. Öffentliche Spitäler erwirtschaften natürlich keinen Gewinn, im Gegensatz jedoch zu den privaten. Der CEO der zur Hirslanden-Gruppe gehörenden Medclinic schreibt in seinem Bericht von 2016: "Growth, profitability and creating shareholder value are certainly major strategic drivers in ensuring the sustainability of our business», Reinvestitionen sind dabei natürlich auch Teil des business plan. Es erstaunt in diesem Kontext, dass der Hauptaktionär ein südafrikanischer Milliardär ist, der überall dort investiert, wo Gewinne zu machen sind, vor allem in der Tabakindustrie [2]. Ist es richtig, dass die Aktionäre eines südafrikanischen Unternehmens Dividenden aus den Erträgen privater Schweizer Spitäler ziehen, wobei Letztere von einer staatlichen Beteiligung für Patienten ohne Privatversicherung profitieren, was ihnen erlaubt, auf der Liste der zugelassenen Spitäler gelistet zu sein und so ihren Kundenstamm ohne zusätzliche Kosten zu erhöhen? Erstaunlich ist ausserdem, dass sich eine dieser Kliniken rühmt, die Hälfte ihrer Ärzte in der Kardiologie und der Herzchirurgie verfügten über einen akademischen Titel [3]. Da liesse sich vermuten, dass es für einen Professor rentabler ist, in einer Privatklinik zu arbeiten als an einem Universitätsspital.

Die Initiative Pro Service Public wurde mit grosser Mehrheit abgelehnt. Letztendlich scheint die Schweizer Bevölkerung doch der Meinung gewesen zu sein, der Service public sei nicht ganz so schlecht und es sich nicht lohne, sich auf ungewisse Abenteuer einzulassen. Die Schweizer sind momentan auch zufrieden mit ihrem Gesundheitssystem und eine Initiative zu Veränderungen in dessen Betrieb dürfte gegenwärtig wohl kaum Chancen haben. Wir müssen jedoch wachsam bleiben, denn es könnte zu ethischen Problemen kommen, wenn die sozialen Aspekte unseres auf Qualität und Solidarität basierten Gesundheitssystems, dessen Kosten für einen grossen Teil der Gesellschaft nicht mehr finanzierbar sind, aus den Augen verloren gehen und unverhältnismässige Gewinne daraus gezogen werden. Riskieren wir keine künftige Initiative «Pro Service Public in der Medizin»! 